\title{
Bokashi como adubação de cobertura: um estudo de caso no desenvolvimento do cultivo de pepino Aodai (Cucumis sativus L.) em uma propriedade rural
}

\section{Bokashi as top dressing: a case study in the development of cucumber Aodai (Cucumis sativus $\mathbf{L}$.) cultivation in a rural property}

Jéssica Rodrigues dos SANTOS ${ }^{1}$; Esmeralda Aparecida Porto LOPES²; João Pedro Ferreira BARBOSA ${ }^{3}$; Jessica Maria Bezerra do ESPIRITO SANTO ${ }^{4}$.

(1) Graduanda do curso Licenciatura em Ciências Biológicas / Universidade Estadual de Alagoas, Campus I/Arapiraca

ORCID: 0000-0003-0729-8164

* E-mail: jessicallais2014@gmail.com

(2) Professora Adjunta da Universidade Estadual de Alagoas, Campus I/Arapiraca

(3) ORCID: 0000-0003-3765-0712

*E-mail: esmeralda.porto@uneal.edu.br

(3) Mestrando do curso de Pós-Graduação em Agronomia (Produção Vegetal) / Universidade Federal de Alagoas, Campus de Engenharias e Ciências Agrárias, Rio Largo

*E-mail: barbosapedro112@gmail.com

ORCID: 0000-0001-9689-435X

(4) Graduanda do curso de Licenciatura em Ciências Biológicas/ Universidade Estadual de Alagoas, Campus I/Arapiraca

*E-mail: jessik_maria@hotmail.com ORCID: 0000-0003-4178-1934

Resumo - O pepino (Cucumis sativus L.) tem grande importância econômica e social dentro do agronegócio de hortaliças no Brasil, gera renda e muitos empregos diretos e indiretos. $\mathrm{Na}$ produção de alimentos um manejo adequado do solo deve garantir a produtividade e sustentabilidade ambiental. Uma das alternativas é a utilização de resíduos orgânicos no desenvolvimento das culturas, diminuindo a dependência de adubos minerais. O objetivo do presente trabalho foi analisar o desenvolvimento do cultivo de pepino tipo Aodai sob o uso de biofertilizante em adubação de cobertura. $\mathrm{O}$ experimento foi em campo em uma propriedade rural de agricultura familiar localizada no Município de São Sebastião - AL. Foi utilizado o delineamento experimental em blocos ao acaso com dois tratamentos: dose $50 \mathrm{ml} / \mathrm{planta}$ de bokashi $(4,7 \%)$ e $50 \mathrm{ml} /$ planta de água (testemunha) e quatro repetições. Cada parcela apresentou uma área útil de $62,4 \mathrm{~m}^{2}$, totalizando 39 plantas espaçadas de 1,00 x 1,20 m. Para as avaliações foram utilizadas 3 plantas por parcela de cada tratamento. As variáveis analisadas foram: número de frutos por planta $(\mathrm{NF} / \mathrm{P})$, peso de fruto por planta $(\mathrm{PF} / \mathrm{P})$, número de frutos por hectare $(\mathrm{NF} / \mathrm{ha})$ peso de fruto por hectare $(\mathrm{PF} / \mathrm{ha})$. Os dados foram submetidos à análise de variância pelo Teste $\mathrm{F}(\mathrm{p}<0,05)$. Não houve significância entre os tratamentos pelo teste $\mathrm{F}$ em nenhuma das variáveis analisadas, no entanto, apesar de não haver diferenças significativas entre os tratamentos, o biofertilizante promoveu um incremento de $33,7 \%$ no NF/P, 31,46\% no $\mathrm{PF} / \mathrm{P}, 34,43 \%$ no NF/ha e $20,58 \%$ no $\mathrm{PF} /$ ha no pepino quando comparados à testemunha.

Palavras-chave: Biofertilizante. Hortaliça. Produtividade.

Abstract - Cucumber (Cucumis sativus L.) has great economic and social importance within the vegetable agribusiness in Brazil, generates income and many direct and indirect jobs. In food production, proper soil management must ensure productivity and environmental sustainability. One of the alternatives is the use of organic residues in the development of crops, 
reducing dependence on mineral fertilizers. The objective of the present work was to analyze the development of the cultivation of Aodai type cucumber under the use of biofertilizer in topdressing. The experiment was carried out in the field on a rural family farm located in the city of São Sebastião - AL. A randomized block design with two treatments was used: dose 50 $\mathrm{ml} /$ plant of bokashi $(4.7 \%$ ) and $50 \mathrm{ml} /$ plant of water (control) and four replications. Each plot had a useful area of $62.4 \mathrm{~m} 2$, totaling 39 plants spaced $1.00 \times 1.20 \mathrm{~m}$. For the evaluations, 3 plants per plot of each treatment were used. The variables analyzed were: number of fruits per plant $(\mathrm{NF} / \mathrm{P})$, weight of fruit per plant $(\mathrm{PF} / \mathrm{P})$, number of fruits per hectare $(\mathrm{NF} / \mathrm{ha})$ weight of fruit per hectare $(\mathrm{PF} / \mathrm{ha})$. Data were subjected to analysis of variance using the $\mathrm{F}$ test $(\mathrm{p}<0.05)$. There was no significance between the treatments by the F test in any of the analyzed variables, however, although there were no significant differences between the treatments, the biofertilizer promoted an increase of $33.7 \%$ in NF/P, $31.46 \%$ in $\mathrm{PF} / \mathrm{P}, 34.43 \%$ in NF/ha and $20.58 \%$ in $\mathrm{PF} / \mathrm{ha}$ in cucumber when compared to the control.

Keywords: Biofertilizer. Productivity. Vegetables.

\section{Introdução}

O pepino (Cucumis sativus) é uma importante hortaliça- fruto da família Cucurbitácea, originária da Índia, de grande valor nutritivo e propriedades medicinais, destacando-se pela sua grande importância econômica e social dentro do agronegócio de hortaliças no Brasil, pois além do valor comercial, gera muitos empregos diretos e indiretos, desde o cultivo até a sua comercialização em todo o mundo, principalmente na China (ANTONIO et al., 2017).

O Brasil produz acima de 200 mil toneladas, cabendo a região Sudeste a produção de mais da metade desse total (CARVALHO et al., 2013). A produção em Alagoas é relativamente pequena, visto que no total de 7.733 estabelecimentos com horticultura, apenas $0,34 \%$ destes estabelecimentos produzem pepino (IBGE, 2017). Por esse motivo não costumam figurar nas estatísticas de produção nacional de forma mais significativa.

Pode ser cultivado na maior parte de tipos de solos, temperaturas entre 20 e $30^{\circ} \mathrm{C}$. Necessita de distribuição regular de água durante todo o ciclo e cerca da metade do fertilizante é aplicado no solo antes do plantio ou sobre as faixas na hora do plantio. A recomendação é que no plantio sejam aplicados $30 \%$ do adubo nitrogenado, $40 \%$ do potássio e toda a dose de fósforo recomendada. As doses restantes de $\mathrm{N}$ e $\mathrm{K}$ devem ser aplicadas em três vezes em cobertura, sendo a primeira 20 dias após a semeadura ou 15 dias após o transplante e as outras duas doses com intervalos de 20 dias uma da outra (CARVALHO et al., 2013).

A utilização da adubação mineral tem elevado o custo de produção, pois os preços dos principais fertilizantes tiveram um aumento de mais de 200\% (LOPES, 1998). A produção 
nacional atende somente a $2 \%$ da produção mundial, ou seja, temos que importar mais de dois terços de nossas necessidades de fertilizantes minerais (CELLA; ROSSI, 2010).

Além disso a dependência excessiva de fertilizantes químicos têm levado a perda gradual de nutrientes do solo, promovendo um ciclo vicioso, em que as plantações futuras necessitam de uma maior quantidade de fertilizantes (COELHO JUNIOR, 2005). Tornando a sua utilização restrita ou mesmo impraticável por agricultores familiares. No entanto, os efeitos dessa prática vão muito além disso e podem representar um grande perigo para o meio ambiente, impactando a qualidade do solo, água, ar e até mesmo a saúde humana (BITTENCOURT, 2009).

Confrontando-se a este manejo convencional, em que o solo é considerado apenas suporte físico para as plantas, baseado em pacotes químicos destinados a nutrir as plantas, surgem novas práticas de manejo que visam a conservação do solo e da água e priorizam a diminuição do uso de insumos externos e que ajudam a manter a saúde do solo. Dentre as alternativas para reposição dos nutrientes ao solo, os biofertilizantes são adubos orgânicos que são submetidos ao processo de fermentação, e podem ter sua origem de qualquer tipo de matéria orgânica.

Entre os grupos de biofertilizantes, o bokashi é resultante de um método de compostagem baseado na adição de uma solução líquida de microrganismos efetivos, que são bactérias anaeróbicas e fermentos do ácido láctico (VICENTE et al., 2020). O bokashi, além de servir como fonte de nutrientes para as plantas, tem a função muito importante de estimular o aumento e a diversidade de organismos que vivem no solo e por ter quantidade balanceada de macro e micronutrientes, o seu uso favorece a boa nutrição das plantas. (SIQUEIRA; SIQUEIRA, 2013)

Considerando que há um mercado com consumidores com forte preocupação ambiental e interessados em produtos mais saudáveis, práticas agrícolas com potencial para substituir os fertilizantes sintéticos tem aumentado significativamente nos últimos anos e verifica-se que existem poucos trabalhos utilizando bokashi em cobertura no pepino. Contudo, o objetivo desta pesquisa foi analisar o desenvolvimento do cultivo de pepino tipo Aodai sob o uso de biofertilizante tipo bokashi como adubação de cobertura.

\section{Material e métodos}


A pesquisa foi realizada no povoado Perna Gorda em uma propriedade rural de agricultura familiar, localizada na zona rural do município de São Sebastião - Alagoas, com Latitude: 9० 94'84" Sul, Longitude: 36 53'47” Oeste. O clima é predominante do tipo tropical chuvoso com verão seco, o período chuvoso começa no outono tendo início em fevereiro e término em outubro. A precipitação média anual é de 1.634.2 mm (MME, 2005).

A análise química e física do solo após a calagem foi quantificada pelo laboratório Central Analítica em Maceió- AL, que apresentou os resultados verificados na tabela 1.

As características físicas observadas na fase de pré-instalação revelaram que a classe textual do solo é franco arenosa, com alta proporção de areia (58\%), caracterizando uma alta porosidade e permeabilidade, além de baixa capacidade de retenção de água. De acordo Carvalho et al. (2013), o solo adequado para o cultivo de pepino é o de textura média (mínimo de $25 \%$ de argila), leve, profundos, férteis e bem drenado e com alto ter de matéria orgânica.

Tabela 1. Características físicas ${ }^{1}$ e químicas ${ }^{1}$ do solo utilizado no experimento.

\begin{tabular}{|c|c|c|c|c|c|c|c|c|c|c|c|}
\hline \multicolumn{12}{|c|}{ Características químicas } \\
\hline Solo & $\mathrm{pH}$ & $\mathrm{P}$ & $\mathrm{K}$ & $\mathrm{Na}$ & $\mathrm{Mg}$ & $\mathrm{Ca}$ & $\mathrm{Al}$ & $\mathrm{H}$ & $\bar{S}$ & CTC & $\mathrm{V}$ \\
\hline Profundidade $(\mathrm{cm})$ & $\mathrm{H}_{2} \mathrm{O}$ & \multicolumn{3}{|c|}{$\mathrm{ppm}$} & \multicolumn{6}{|c|}{ meq/100ml } & $\%$ \\
\hline $0-20$ & 5,3 & 10 & 33 & 31 & 1,5 & 1,3 & 0,41 & 3,2 & 3,0 & 3,43 & 48,5 \\
\hline \multicolumn{12}{|c|}{ Características físicas } \\
\hline $\begin{array}{l}\text { Solo } \\
\text { Profundidade (cm) }\end{array}$ & \multicolumn{2}{|c|}{ Areia grossa } & \multicolumn{2}{|c|}{ Areia Fina } & \multicolumn{2}{|c|}{ Areia total } & \multicolumn{2}{|c|}{ Silte } & Argila & \multicolumn{2}{|c|}{ Classe textural } \\
\hline $0-20$ & \multicolumn{2}{|c|}{318} & \multicolumn{2}{|c|}{262} & \multicolumn{2}{|c|}{580} & \multicolumn{2}{|c|}{255} & 166 & \multicolumn{2}{|c|}{ FA* } \\
\hline
\end{tabular}

\section{Produção do biofertilizante tipo Bokashi}

O biofertilizante foi produzido a partir da captura de microrganismos eficazes em um fragmento de mata próximo à área do experimento. Para isso foi utilizado $700 \mathrm{~g}$ de arroz que foi colocado em bandejas e cobertos por uma fina tela de proteção, sendo depositadas no solo e cobertas com uma camada de serrapilheira por 15 dias.

Os microrganismos presentes no arroz que apresentaram as colorações rosada, azulada, amarelada e alaranjada foram isolados e ativados em uma mistura de $1 \mathrm{~kg}$ de açúcar demerara mais 10 litros de água filtrada. Logo após foram distribuídos em 5 garrafas PET de 2 litros e mantidos hermeticamente fechadas, sendo que a cada dois dias, as garrafas eram abertas para a 
liberação de gases produzidos pelo processo de fermentação (SIQUEIRA; SIQUEIRA, 2013). Quando a produção de gás encerrou, o bokashi foi diluído na concentração de 4,7\%.

\section{Condução do Plantio de Pepino}

A área total cedida pelo agricultor foi de $295,8 \mathrm{~m}^{2}$. O solo foi corrigido com calcário 2t/ha para reduzir a acidez. O delineamento experimental foi em blocos ao acaso com dois tratamentos: dose $50 \mathrm{ml} /$ planta de bokashi $(4,7 \%)$ e $50 \mathrm{ml} /$ planta de água (testemunha) com quatro repetições. As parcelas apresentaram uma área útil de $62,4 \mathrm{~m}^{2}$ (13 metros de comprimento por 4,80 metros de largura). Antes do plantio foi feito uma adubação com $200 \mathrm{~g} / \mathrm{m}^{2} \mathrm{de}$ esterco bovino e $100 \mathrm{~g} / \mathrm{m}^{2}$ de cinzas.

As sementes do pepino Aodai (Cucumis sativus) marca Topseed foram adquiridas em lojas comerciais e plantadas espaçadas 1,00 m entre plantas x 1,20 m entre linhas, totalizando 39 plantas por parcela, que foram manejadas de forma rasteira. Foram identificadas com placas um total de 6 plantas por parcela, sendo 3 de cada tratamento.

Após o $20^{\circ}$ (vigésimo) dia da semeadura, iniciou-se a aplicação do bokashi via adubação de cobertura, realizada no período do final da tarde utilizando um pulverizador costal e mantida semanalmente até a última colheita. A irrigação foi feita todos os dias através de sistema de gotejamento, sendo que em dias mais quentes eram feitas duas vezes ao dia. Foram feitas duas colheitas aos 50 e 73 dias após a semeadura. O controle das ervas resistentes foi de forma manual e aos 43 dias após o plantio foi aplicado uma única dose do herbicida glifosato.

Todas as variáveis analisadas como: número de frutos por planta (NF/P), peso de frutos por planta $(\mathrm{PF} / \mathrm{P})$, número de frutos por hectare $(\mathrm{NF} / \mathrm{ha})$ e peso de frutos por hectare $(\mathrm{PF} / \mathrm{ha})$ foram resultados da primeira e segunda colheita. Os dados obtidos foram transformados em $\sqrt{x}+1$ e submetidos à análise de variância pelo teste $\mathrm{F}(\mathrm{p}<0,05)$ no software SISVAR 5,6.

\section{Resultados e Discussão}

O ciclo total do cultivo foi de 73 dias, sendo a primeira e segunda colheita aos $50^{\circ}$ (quinquagésimo) dias e $73^{\circ}$ (septuagésimo terceiro) dias após a semeadura, respectivamente. Os resultados com a análise estatística do número de frutos por planta, peso de fruto por planta, número de frutos por hectare e peso de frutos por hectare em função da utilização do biofertilizante, estão na tabela 2 . 
Evidenciou-se através da tabela 2, que a saturação (V) por bases $(48,5,7 \%)$ não é a recomendada para a cultura do pepino, que é aproximadamente 75\% (CARVALHO et al., 2013). Contudo, é possível constatar através dos resultados obtidos da análise de solo, que o ambiente experimental, tanto fisicamente quanto quimicamente apresentou limitações para suprir nutrientes à cultura de pepino.

Tabela 2. Número de frutos por planta (NF/P), peso de frutos por planta (PF/P), número de frutos por hectare $(\mathrm{NF} / \mathrm{ha})$, peso de frutos por hectare $(\mathrm{PF} / \mathrm{ha})$ do pepino Aodai (Cucumis sativus) após aplicação do Bokashi como adubação de cobertura.

\begin{tabular}{ccccc}
\hline Tratamentos & $\begin{array}{c}\text { NF/P } \\
\text { (un) }\end{array}$ & $\begin{array}{c}\text { PF/P } \\
(\mathrm{kg})\end{array}$ & $\begin{array}{c}\text { NF/ha } \\
\text { (un) }\end{array}$ & $\begin{array}{c}\text { PF/ha } \\
(\mathrm{kg})\end{array}$ \\
\hline \hline Bokashi 4,7\% & $3,66 \mathrm{a}$ & $1,17 \mathrm{a}$ & $61,111 \mathrm{a}$ & $17,907 \mathrm{a}$ \\
Sem Bokashi & $2,74 \mathrm{a}$ & $0,89 \mathrm{a}$ & $45,833 \mathrm{a}$ & $14,851 \mathrm{a}$ \\
Médias & 3,20 & 1,030 & 53,472 & 16,275 \\
\hline CV (\%) & 20,57 & 13,95 & 28,08 & 26,74 \\
\hline
\end{tabular}

Médias seguidas pela mesma letra na mesma coluna não diferem estatisticamente entre si pelo teste de $\mathrm{F}(\mathrm{p}<0,05)$. Dados transformados em $\sqrt{x}+1$

Observa-se que o uso de biofertilizante tipo bokashi a 4,7\% não diferiu estatisticamente no número de frutos por planta $(\mathrm{NF} / \mathrm{P})$, peso dos frutos por planta $(\mathrm{PF} / \mathrm{P})$, número de frutos por hectare $(\mathrm{NF} / \mathrm{ha})$ e no peso de frutos por hectare $(\mathrm{PF} / \mathrm{ha})$ em comparação ao tratamento sem bokashi. No entanto, apesar de não haver diferenças significativas entre os tratamentos, o bokashi promoveu um incremento de $33,7 \%$ no NF/P, $31,46 \%$ no $\mathrm{PF} / \mathrm{P}, 34,43 \%$ no NF/ha e 20,58\% no PF/ha quando comparados à testemunha.

Embora os biofertilizantes demonstrem melhoria no desempenho de culturas quando utilizados conforme as recomendações, deve-se levar em consideração vários fatores como: conhecer as características físicas e químicas do solo se são adequados para a cultura, visto que, solos arenosos e de baixa fertilidade natural, apresentam baixa capacidade de retenção de água e nutrientes, aumentando com isso o potencial de perda de nutriente, fato que pode ter ocorrido neste estudo, visto que foi constatado através dos resultados obtidos da análise de solo, que o mesmo não era adequado para o cultivo de pepino por apresentar uma alta proporção de areia e uma baixa saturação por bases (tabela 1), demonstrando com isso as dificuldades vivenciadas pela agricultura familiar, que embora apresente potencial econômico e social considerável no Brasil, na região Nordeste, uma grande proporção de agricultores é exercida por produtores pouco qualificados e tecnificados, revelando o quanto é necessário para a atividade, a oferta de políticas públicas de assistência técnica e extensão rural. 
Desempenhos baixos com aplicação de biofertilizantes também foi observado por Silva et al. (2007), que avaliando diferentes tipos de biofertilizantes líquidos (Agrobom, Fermentado de Rúmem e Vairo) via fertirrigação na cultura do melão amarelo no semiárido Pernambucano, mostraram que o incremento foi de apenas $2,9 \%$ e $11,8 \%$ na produtividade do melão quando foi aplicado o Fermentado de Rúmem e Vairo, respectivamente, quando comparados à testemunha. Já o biofertilizante Agrobom promoveu um decréscimo na produtividade de $8,04 \%$.

A eficácia da aplicação do biofertilizante na produtividade foram demonstrados por Galbiatti et al. (2011), que estudaram o desenvolvimento da cultura de feijoeiro (Phaseolus vulgaris L.) com e sem a aplicação de biofertilizante (efluente de biodigestor à base de esterco bovino) obtendo as melhores médias de massa seca das folhas, caule e pecíolo, parte aérea, e a área foliar nos tratamentos que receberam biofertilizante. Antonio et al. (2017) verificaram ao avaliar diferentes concentrações $(2 \%, 5 \%, 10 \%$ e $15 \%)$ de biofertilizante a base de esterco bovino fermentado como adubação complementar no pepino híbrido Kouki F1 (tipo japonês), via fertirrigação, concluíram que a concentração de $2 \%$ e $15 \%$ promoveram um maior número de F/P e NF/há quando comparada a $10 \%$.

Para que um biofertilizante expresse sua capacidade de nutrir é preciso que se faça um trabalho de melhoria na fertilidade do solo por meio do uso de compostos e resíduos de vegetais, o que não se consegue com pouco tempo. Alguns estudos têm mostrado que este resultado só é conseguido com a união de várias práticas agrícolas e não somente com aplicações de produtos isolados. Aliado a isso, os biofertilizantes seriam então usados como fonte imediata de nutrientes, complementando o que existe no solo e a demanda da planta (SILVA et al., 2007). O Bokashi além de ser utilizado na revitalização do solo, restaurando as condições físicoquímicas e microbiológicas, ainda liberam no ambiente alguns compostos que aumentam a resistência das plantas aos insetos e doenças, contribuindo para uma maior autonomia dos agricultores familiares.

\section{Conclusões}

O ambiente experimental, tanto fisicamente quanto quimicamente apresentou limitações para suprir nutrientes à cultura de pepino.

O bokashi como adubação de cobertura na concentração de 4,7\% não influenciou no número de frutos por planta $(\mathrm{NF} / \mathrm{P})$, peso dos frutos por planta $(\mathrm{PF} / \mathrm{P})$, número de frutos por 
hectare (NF/ha) e no peso de frutos por hectare (PF/ha); no entanto, apesar de não haver diferenças significativas entre os tratamentos, o biofertilizante promoveu um incremento de $33,7 \%$ no NF/P, 31,46\% no PF/P, 34,43\% no NF/ha e 20,58\% no PF/ha no pepino do tipo Aodai quando comparados à testemunha.

\section{Agradecimentos}

Agradeço a professora Dra. Esmeralda Porto Lopes, orientadora deste estudo.

Ao Sr. Wilson Vagetti e Sra. Luzinete Vagetti por tornar possível a realização deste trabalho em sua propriedade rural. Ao agrônomo Rony Holanda por tirar nossas dúvidas técnicas. Aos companheiros de ajuda em campo, Leomárcio, Jadson, Jéssica Maria e Joyce.

\section{Conflitos de interesse}

Os autores desse manuscrito não declararam conflito de interesses.

\section{Referências}

ANTONIO, C. I.; CARDOSO, O M.; BERNI, F. R.; KANO, C. Uso de biofertilizante na adubação complementar do pepino sob ambiente protegido. Manaus: Embrapa Amazônia Oriental, 2017. (Boletim de Pesquisa e Desenvolvimento).

BITTENCOURT, V. L. Impactos da agricultura no meio-ambiente: principais tendências e desafios. Economia \& Tecnologia, v. 5, v. 18, p. 133-146, 2009.

BRASIL. Instituto Brasileiro de Geografia e Estatística. Censo Agropecuário 2017.

Disponível em:

$<$ https://www.ibge.gov.br/estatisticas/economicas/agricultura-e-pecuaria/21814-2017-censoagropecuario.html?=\&t=resultados $>$. Acesso em 22 de outubro de 2021.

BRASIL. Ministério de Minas e Energia. Diagnóstico do município de São Sebastião. 2005. Disponível em:

<https://rigeo.cprm.gov.br/jspui/bitstream/doc/15346/1/rel_cadastros_sao_sebastiao.pdf>. Acesso em 18 de outubro de 2021.

CARVALHO, A. D. F.; AMARO, G. B.; LOPES, J. F.; VILELA, N. J.; MICHEREFF FILHO, M.; ANDRADE, R. A cultura do pepino. Brasília: Embrapa Hortaliças, 2013. (Circular técnica 113). 
CELLA, D.; LIMA, M. C. R. Análise do mercado de fertilizantes no Brasil. Revista Interface Tecnológica, v. 7, n. 1, p. 41-50, 2010.

COELHO JUNIOR, E. V. A modernização da agricultura brasileira e alguns de seus aspectos socioambientais: o caso da região de Goianápolis-GO. Dissertação (Mestrado em Sociologia) - Faculdade de Ciências Humanas, Universidade Federal de Goiás. Goiânia, p. 156. 2005.

FERREIRA, D. F. Sisvar: a computer analysis system to fixed effects split plot type designs. Revista Brasileira de Biometria, v. 37, n. 4, p. 529-535, 2019.

FINATTO, J.; ALTMAYER, T.; MARTINI, M. C.; RODRIGUES, M.; BASSO, V.; HOEHNE, L. A importância da utilização da adubação orgânica na agricultura. Revista Destaques Acadêmicos, v. 5, n. 4, p. 85-93, 2013.

GALBIATTI, A. J.; SILVA, G. F.; FRANCO, F. C.; CARAMELO, D. A. Desenvolvimento do feijoeiro sob o uso de biofertilizante e adubação mineral. Engenharia Agrícola, v. 31, n. 1, p. 167-177, 2011.

LOPES, A. S. Manual de fertilidade do solo. Piracicaba: Potafos, 1998.

SILVA, A. F.; PINTO, J. M.; FRANÇA, C. R. R. S.; FERNANDES, S. C.; GOMES, T. C. A.; SILVA, M. S. L.; MATOS, A. N. B. Preparo e uso de biofertilizantes líquidos. Petrolina: Embrapa Semi-Árido, 2007. (Comunicado Técnico 130).

SILVA, A. C. M. Biofertilizantes: estudo de opinião, tendência das pesquisas e legislação brasileira. Dissertação (Mestrado em Ciências Ambientais) - Universidade de Brasília. Brasília, p. 70, 2021.

SIQUEIRA, A. P. P.; SIQUEIRA, M. F. B. Adubo orgânico fermentado. Niterói: Programa Rio Rural, 2013.

VICENTE, N. F. P.; MARAFELI, E. A. M.; OLIVEIRA, J. A. C.; TOMITA, J. L. C.; PICCOLI, R. H. Uma revisão bibliográfica sobre bokashi dos últimos 20 anos. Research, Society and Development, v. 9, n. 10, p. 1-44, 2020. 